Review

\title{
Cell competition and tumorigenesis in the imaginal discs of Drosophila
}

\author{
Ginés Morata*, Manuel Calleja \\ Centro de Biología Molecular, CSIC-UAM, Nicolas Cabrera 1, Madrid, 28049, Spain
}

\section{A R T I C L E I N F O}

\section{Keywords:}

Cell competition

Tumorigenesis

JNK

Apoptosis

\begin{abstract}
A B S T R A C T
Cancer is a major health issue and the object of investigations in thousands of laboratories all over the world. Most of cancer research is being carried out in in vitro systems or in animal models, generally mice or rats. However, the discovery of the high degree of genetic identity among metazoans has prompted investigation in organisms like Drosophila, on the idea that the genetic basis of cancer in flies and humans may have many aspects in common. Moreover, the sophisticated genetic methodology of Drosophila offers operational advantages and allows experimental approaches inaccessible in other species.

Cell competition is a cell-quality control process that aims to identifying and subsequently removing cells within animal tissues that are unfit, abnormal or aberrant, and that may compromise the fitness or the viability of the organism.

It was originally described in Drosophila imaginal discs but later work has shown it occurs in mammalian tissues where it fulfils similar roles. One aspect of the surveillance role of cell competition is to eliminate oncogenic cells that may appear during development or the life of an organism. In this review we have focussed on the work on Drosophila imaginal discs relating cell competition and tumorigenic processes. We briefly discuss related work in mammalian tissues.
\end{abstract}

\section{Drosophila and Cancer}

The fruitfly Drosophila has been a major object of genetic research for a long time, since the beginning of the XX century. The entire life cycle lasts 10-11 days in optimal conditions. After about $24 \mathrm{~h}$ of embryonic development, there are three larval stages before pupariation and metamorphosis, after which the new adult emerges. The larvae contain inside the precursors of the adult structures, called imaginal discs, which are named after the particular adult structure, wing, leg, eye-antenna, etc., they will eventually form. The imaginal discs are easily identified and genetically manipulated during the larval period and are a convenient system to study tumour overgrowths [1-3].

Cancer may appear spontaneously in flies [4], but most of the information about tumorigenesis in Drosophila derives from the identification and analysis of a number of genes whose mutations cause tumorigenesis (reviewed in [2], Table 1). These mutations are lethal but allow larval development; the mutant larvae grow very large and contain big tumour overgrowths, usually affecting the imaginal discs and the nervous system.

Those genes are usually referred to as tumour-suppressing genes (TSG) based on that their loss of function causes tumour development. However, they are involved with a number of biological processes, e.g. apico-basal polarity, endocytosis, cell identity, etc. not directly related with growth control. How defects in these processes cause tumorigenesis is not entirely clear; it is likely that it is a secondary consequence of the principal defect.

The number of TSGs is large (see Flybase, https://www.sdbonline. org/sites/fly/aignfam/tumorsup.htm), thus in this review we will consider some whose mutations cause neoplastic transformations and in which cell competition has been shown to play a role. We will discuss results obtained with lethal giant larvae (lgl), scribble (scrib), erupted (ept), Rab5 and polyhomeotic (ph). Their tumorigenic effect has been characterised in the imaginal discs, usually the eye or the wing disc.

A significant feature of the TSG mutant cells is that, unlike normal cells, they can proliferate indefinitely. The proliferation rate is not higher than in normal cells but the mutant cells remain proliferating indefinitely until the larva dies [5-7]. This feature of not responding to stop growth signals is one of the distinctive tumorigenic features.

A detailed study of the morphological and developmental alterations of homozygous larvae and discs mutant for TSGs is still lacking, but a common feature of $l g l$, rab5 and erupted discs is that they show high levels of apoptosis and of JNK activity [7]; Calleja, unpublished), although their involvement of these factors in the development of the tumours not been studied. One of the known targets of JNK is the

\footnotetext{
* Corresponding author.

E-mail address: gmorata@cbm.csic.es (G. Morata).
} 
Table 1

A sample of Drosophila genes and their human homologs whose mutations are associated with tumour transformations both in humans and in flies. These genes are implicated in a variety of functions, as indicated in the Table. It is not clear how the defects in any of these functions are translated into a tumorigenic transformation.

\begin{tabular}{|c|c|c|c|}
\hline function & D.m. genes & human genes & References \\
\hline \multirow[t]{3}{*}{ Cell signaling } & $h h$ & Shh/Dhh/Ihh & Lin et al., 2016 \\
\hline & $w g$ & Wnts & $\begin{array}{l}\text { Clevers and Nusse, } \\
2012\end{array}$ \\
\hline & $\begin{array}{l}J N K \text { pathway } \\
\text { genes }\end{array}$ & JNK & Chen, 2012 \\
\hline \multirow[t]{3}{*}{ Insulin } & Pten & PTEN & Li et al., 1997 \\
\hline & $t s c 1$ & TSC1 & Pan et al., 2004 \\
\hline & gigas & TSC2 & Pan et al., 2004 \\
\hline \multirow[t]{4}{*}{ Size control } & Hippo & Hippo & Harvey et al., 2013 \\
\hline & sav & hSAV1 & Luo et al., 2011 \\
\hline & $w t s$ (lats) & $\begin{array}{l}\text { LATS1 (h-warts) } \\
\text { LATS2/(HsLATS2) }\end{array}$ & $\begin{array}{l}\text { Deng et al., 2017/Luo } \\
\text { et al., } 2014\end{array}$ \\
\hline & Brat & TRIM3 & Chen et al., 2014 \\
\hline \multirow[t]{5}{*}{ Endocytosis } & $a v l^{*}$ & STX7 & $\begin{array}{l}\text { Strömberg et al., } \\
2009\end{array}$ \\
\hline & Rab5* & RAB5 & Igarashi et al., 2017 \\
\hline & $v p s 25^{*}$ & VPS25 & Menut et al 2007 \\
\hline & $e p t^{*}$ & TSG101 & $\begin{array}{l}\text { Xu And Zheng et al., } \\
2019\end{array}$ \\
\hline & snr1 & SMARCB1 & Pawel et al., 2018 \\
\hline \multirow[t]{3}{*}{ Cell polarity } & scrib* & hScrib & Ouyang et al., 2010 \\
\hline & $d l g^{*}$ & $\mathrm{hDlg} 1 / 2 / 3 / 4$ & Mori et al., 1998/ \\
\hline & $l(2) g l^{*}$ & Hugl1/2 & Russ et al., 2012 \\
\hline Notch signalling & $N$ & $\mathrm{~N}$ & $\begin{array}{l}\text { Nowell and Radtke, } \\
2017\end{array}$ \\
\hline \multirow[t]{2}{*}{ Polycomb } & ph & PHC1 & Martinez et al., 2009 \\
\hline & $l(3) m b t$ & L3MBT1 & Koga et al., 1999 \\
\hline
\end{tabular}

Matrix metalloprotease 1 (Mmp1), involved in breakdown of the extracellular matrix, thus favouring cell invasion and possibly metastasis. In the case of the $l g l$ mutant larvae, we have shown that Mmp1 is required for the fusion of imaginal discs [7].

\section{Cell competition}

Cell interactions are widespread in animal tissues and play key roles in processes like pattern formation, morphogenesis and cell differentiation. Among those, cell competition (CC) is a special kind of cell-cell interaction that plays an important role in tissue homeostasis by removing unfit or undesirable cells.

Animal tissues usually contain millions of cells, making it inevitable the appearance of unfit/abnormal/aberrant cells generated by somatic mutation [8] or other causes (reviewed in [3]. This calls for the existence of a mechanism to remove those cells that are not appropriate for the resident tissue and that may compromise the fitness or the survival of the organism.

The cell competition (CC) phenomenon fits the above description; it is a cell-quality control mechanism responsible for the elimination of cells that are weaker or are not well integrated within the population. It was originally reported in the wing disc of Drosophila [9] when investigating the proliferation rate of cells lacking one dose of a Minute gene. The Minute genes constitute a class of genes encoding ribosomal proteins [10]. Flies with only one dose of Minute are viable but show retarded development, likely due to reduction in protein synthesis [11]. The key observation was that viable but slow proliferating cells heterozygous for a Minute mutation were eliminated by interactions with more rapidly dividing neighbours.
Later work extended this phenomenon to other situations; viable cells with low levels of $d M y c$ (the Drosophila homolog of the mammalian oncogene $M y c$ ) or with low activity of the insulin pathway are eliminated when surrounded by cells with higher $d M y c$ or metabolic activity [12,13]. Interestingly, cells with wildtype levels of $d M y c$ are eliminated when confronting others with higher $d M y c$ activity $[14,15]$. This phenomenon, termed "supercompetition", reflects the context-dependence nature of cell competition. Moreover, the fact that higher levels of $d M y c$ generate overproliferation may suggest that supercompetition might be implicated in tumorigenic processes [14-16]. The phenomenon of CC is not limited to developing imaginal discs; recent work from the Piddini group has shown the elimination by apoptosis of sub-optimal cells in the adult intestine [17].

Thus CC describes interactions between two types of viable cells, nowadays referred to as winner and loser, which result in the elimination of the loser. The key aspect of CC is that it is a context-dependant phenomenon: loser cells are themselves viable; it is just their interaction with the winners what causes their demise [18].

The role of CC is not only limited to remove unfit or less metabolically active cells; it also includes cells that, due to incorrect levels of developmental signals [19] or mutations at identity genes [20], are incorrectly specified within the resident tissue. This phenomenon was originally called "morphogenetic apoptosis" and is linked to CC [19]. It is thought to function to ensure the uniform developmental specification of cell populations. The interest of these observations is that they indicate that CC has a function broader than just eliminating unfit or suboptimal cells; it also disposes of cells that may be fit or metabolically active, but that are undesirable for the tissue. This point is relevant when describing the behaviour of oncogenic cells (see below).

A major feature of CC is that it appears to function at short range; the winner/loser cells have to be very close [18,21,22], possibly in physical contact. Another important feature, which is often neglected, is that it occurs between cells that belong to the same identity group. This is especially clear in the wing disc, which is subdivided into an anterior (A) and a posterior (P) compartment: CC does not take place across the A/P border [18], even though A and P compartment cells abut along the border. Presumably, the different identities of $\mathrm{A}$ and $\mathrm{P}$ cells prevent the cell competition interaction. The role in CC of properties associated with cell identity, e.g. cell affinity, cell adhesion, has not been investigated.

Although all the original work was carried out in Drosophila, there is now evidence that CC also functions in mammalian tissues. The mouse Bst mutation behaves as a Minute mutation in Drosophila [23]; it is viable in heterozygous condition but the mutant cells have growth disadvantage when surrounded by normal cells. Furthermore [24], reported that during rat liver regeneration transplanted fetal hepatocytes are able to out compete resident hepatocytes; a phenomenon akin to cell competition.

Moreover, there is strong evidence for CC in mouse development $[25,26]$, demonstrating that during early embryogenesis cells with low levels of $M y c$ are eliminated by interaction with neighbours with higher levels. The removal of these less metabolically active cells is thought to have a positive effect on the fitness of the organism.

A very recent report [27] describes a morphogenetic function of CC during mouse skin development. In the early embryonic stage, slow dividing progenitors (contain lower levels of $M y c n$ ) are eliminated by apoptosis and subsequent engulfment by neighbours with higher proliferation rate. When cell competition is abrogated the stratified structure of the skin becomes abnormal, an indication of the functional relevance of cell competition in normal development.

In conclusion, CC fulfils a general homeostatic role in animal tissues by eliminating viable cells with are undesirable for the resident tissue; they may be less fit or weaker than surrounding cells or may have developmental features that are different from those of the general population. 


\section{Cell competition and cancer}

One especially interesting role of CC is its function as a tumour suppressor mechanism: the removal of malignant/oncogenic cells that may appear during development or the adult life of the organism.

The link between CC and tumorigenesis has been explored by planting in normal tissues isolated oncogenic cells surrounded by normal cells, a situation that mimics the appearance of tumours in mammals. In Drosophila this can be achieved by using recombination techniques $[28,29]$ to generate clones of mutant cells for TSGs.

These clones of oncogenic cells are appropriately labelled and can be distinguished from surrounding normal tissue. This is a type of experiment that can easily be done in Drosophila and that allows the study of the early phases of tumorigenesis, an aspect of the process not easily attained in mammalian systems. Most of the experiments have been carried out in the wing or the eye disc. Typically they consist of generating marked mutant clones for one TSG (lgl, scrib, dlg, rab5, erupted, etc) $[6,30-33]$ during the larval period and then examining the fate and behaviour of the clones several days after clone induction. The general result is that the oncogenic clones are eliminated $48-72 \mathrm{~h}$ after clone initiation (Fig. 2a). As the cells are themselves viable (homozygous mutant larvae develop extensive tumours), their elimination must result from interaction with non-tumour cells, a cell competition scenario. The elimination of oncogenic cells occurs even if they contain high levels of the Ras pathway provided the ras $^{V 12}$ transgene [34], which confer cell proliferation or metabolic levels higher than surrounding normal cells: isolated $\mathrm{lgl}^{-} \mathrm{ras}^{\mathrm{V12}}$ or rab5- $\mathrm{ras}^{\mathrm{V12}}$ clones are eliminated for the tissue $[6,33]$. Apparent exceptions to this rule will be discussed in the next section.

The conclusion for the preceding is that in Drosophila CC functions as a robust tumour-suppressing mechanism able to remove oncogenic cells even if they are physiologically more vigorous that the surrounding normal ones. Thus in a cell competition assay oncogenic cells behave as losers.

An observation of interest is that oncogenic cells are not eliminated in mosaic discs in which, for example, the anterior compartment is normal but the posterior compartment is entirely mutant for $l g l$ or scrib [6] Fig. 2b. Normal and oncogenic cells are abutting along the A/P border and yet there is no CC. It is a clear demonstration that there is no CC across compartment borders, suggesting that the different identities of the A and P compartment cells prevent the CC interaction, possibly because of the differences in cell affinities.

Differences in cell affinities may also be an explanation for the behaviour of clones of cells mutant for polyhomeotics ( $p h$ ), a member of the Polycomb group of genes [35-37], involved in the maintenance of cell identity during development. Unlike mutant cells for the other TSGs investigated, $p h$ clones survive surrounded by non-mutant cells and often form large tumorous overgrowths. Nevertheless, these clones exhibit some special features: while normal clones are usually elongated and integrate with surrounding tissue, $\mathrm{ph}^{-}$clones are usually round and tend to segregate from the normal tissue, growing separately (Fig. 3). It is possible that $p h^{-}$clones acquire cell affinities very different from those of the surrounding cells, in effect preventing the cellcell interaction necessary for cell competition.

The tumour-suppressing function of CC appears to extend to mammalian tissues. Cells mutant for the mammalian homolog of the Drosophila scrib gene have been shown to enter apoptosis in cell cultures when surrounded by normal cells, but not when cultured alone [38], a typical CC scenario. The Fujita group [39] has also shown that transformed epithelial cells are outcompeted by normal epithelial cells and are eliminated from the tissue, revealing a tumour suppressing function that they have termed EDAC (Epithelial Defence Against Cancer).

Work from the Martins group [40] provided a significant observation suggesting that CC may have a tumour suppressing function in mammalian tissues in vivo. During the generation of mature $\mathrm{T}$ cells in the mouse thymus there is a continuous replacement of resident thymocytes by new progenitors coming from the bone marrow. The newcomers appear to be more successful in competing for interleukin-7, which results in the death of the old residents. Martins [40] have shown that the suppression of the influx of new progenitors, and hence of CC, results in indefinite proliferation of the old thymocytes and the appearance of lymphoblastic leukaemia.

Another example of elimination of potentially overgrowing tissue by a mechanism akin to cell competition has been recently reported [41]. These authors generate in the mouse hair follicle epithelium cells with an activated form of $ß$-catenin and observe the formation of outgrowths that are nevertheless promptly eliminated by apoptosis after interaction with neighbour wildtype cells. They obtain a similar result after generating cells containing an oncogenic form of Ras; after an initial overgrowth caused by mutant cells, the follicle returns for normal, presumably because the oncogenic cells have been eliminated.

Considering together the observations from Drosophila and mammalian tissues, the general suggestion is that CC encodes a tumour suppressing function present in all metazoans.

\section{Cell competition, JNK and apoptosis}

There are two major events in the molecular mechanism of CC, 1) the initial comparison between cells, which determines the winner or the loser status, and 2) the activation of the death program in the loser cell.

The mechanism of the cell-cell comparison remains largely unknown. Since cell competition is a short-range phenomenon that may require physical contact, it is likely that it involves comparison of molecules at the cell membranes. One first consequence of the comparison appears to be the modification of the transcriptional activity of the flower gene, which encodes a trans-membrane protein $[42,43]$. There are three different forms of the Flower product, Flower ${ }^{\text {Ubi }}$, Flower $^{\mathrm{LoseA}}$ and Flower ${ }^{\mathrm{LoseB}}$. While Flower ${ }^{\mathrm{Ubi}}$ is expressed in all cells of the disc, Flower ${ }^{\text {LoseA }}$ and Flower ${ }^{\text {LoseB }}$ appear are expressed only in loser cells. The two Lose forms are directly implicated in the process, for their suppression by RNA interference prevents the elimination of the loser cells [43].

The second event, i.e. the activation of the death program by the loser cells is better known, though not completely resolved. Work from the Moreno group has identified the gene azot, that encodes calcium binding protein, as necessary for the elimination of the loser cells [44], but the mechanism by which azot intervenes in the process has not yet been established.

An appealing proposal [45,46] implicates the signalling Spz/Toll module of the innate immune system. Winner cells activate the release of the Spz ligand that acts as a death signal in neighbour loser cells. The process requires Toll and Toll- 8 receptors and eventually leads to the activation of pro-apoptotic genes hid o reaper. Inhibition of Toll activity results in rescue of loser cells. However, the role of Toll in cell competition has been questioned by recent work [47] showing that in axenic (free of pathogens) flies Toll inhibition (by overexpressing Cactus) fails to rescue loser cells in a Minute cell competition scenario. These observations suggest that the effect of Toll on cell competition might be a consequence of its proliferative response to infection.

The first indication of the link between cell competition and the cell death program was reported by Moreno [48] in experiments in the wing disc. Loser Minute heterozygous cells are eliminated by programmed cell death (apoptosis), triggered by the activation of the Jun N-Terminal Kinase (JNK) pathway, the principal stress response factor in Drosophila. Later work has shown that the pro-apoptotic role of JNK is required for the elimination of loser cells in many different cell competition assays, including the elimination of oncogenic cells thus underlying the pivotal role of JNK in CC.

A simplified scheme of the apoptosis program and the position of JNK therein are shown in Fig. 1. JNK exerts autocrine (cell autonomous) pro-apoptotic role and a paracrine (non-cell autonomous) pro- 


\section{Cell proliferation}

\section{JNK maintenance loop}

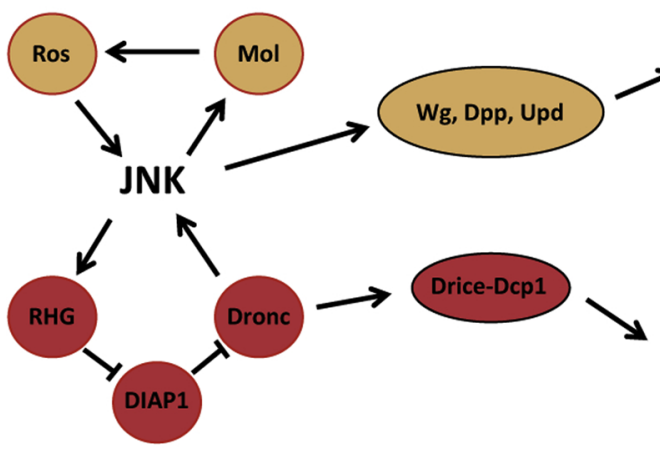

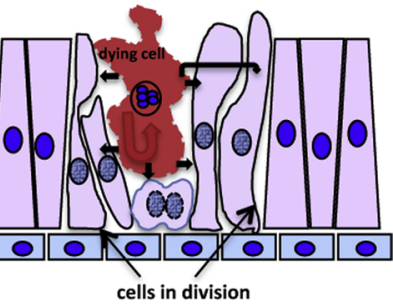

Cell death

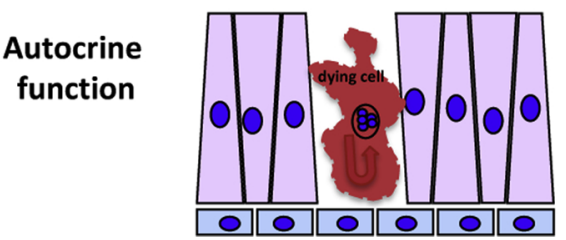

Fig. 1. Simplified scheme of the apoptosis program of Drosophila and the role of JNK in it. Cells expressing JNK activate the function of pro-apoptotic genes, collectively referred to as RHG (for reaper, hid and grim, the principal pro-apoptotic genes) that block the function of the inhibitor of apoptosis diap (the Drosophila inhibitor of apoptosis gene), thus allowing the activation of the apical caspase Dronc and subsequently of the effector caspases Drice and Dcp1 direct responsible for the death of the cell. This reflects the autocrine cell autonomous pro-apoptotic function of JNK. Note that Dronc not only activates Drice and Dcp1 but it also further stimulates JNK activity, thus generating an amplification of the apoptotic levels (Shelkvov and Morata, 2012).

Dying JNK-expressing cells secrete mitogens like Wg, Dpp or Unpaired $[64,65,68]$ that stimulate the proliferation of neighbour cells (illustrated in the upper right side of the figure). This paracrine JNK activity is involved in some

\section{Apoptosis amplification loop}

tumorigenic processes in Drosophila. Note that JNK triggers its own feedback loop via the function of the target moladietz (mol), which stimulates the production of Reactive Oxygen Species (ROS), known to induce JNK (see [51], for a more detailed description). In the majority of the situations cells expressing JNK are short-lived and the paracrine effect is negligible, but when death is delayed or the death machinery is defective the paracrine effect may become predominant.

proliferation function. The paracrine cell proliferation stimulating function of JNK has tumorigenic consequences in certain conditions (see below).

Oncogenic cells normally behave as losers in a CC assay. They express JNK activity previous to their death $[6,30,33,49,50]$ and follow the rules described for Minute CC: activation of the JNK pathway followed by induction of pro-apoptotic genes and subsequently activation of caspases, which results in the death of the cells (Fig. 2a). Importantly, the pro-apoptotic function of JNK is critical for the process, for in its absence oncogenic $\mathrm{lgl}^{-}$or $\mathrm{scrib}^{-}$mutant cells survive [6,51]. Thus the tumour-suppressing role of CC relies on the autocrine function of JNK: oncogenic cells trigger their own demise endogenously activating the JNK-mediated apoptosis program.

A relevant question is how the JNK is activated in the loser cells. a

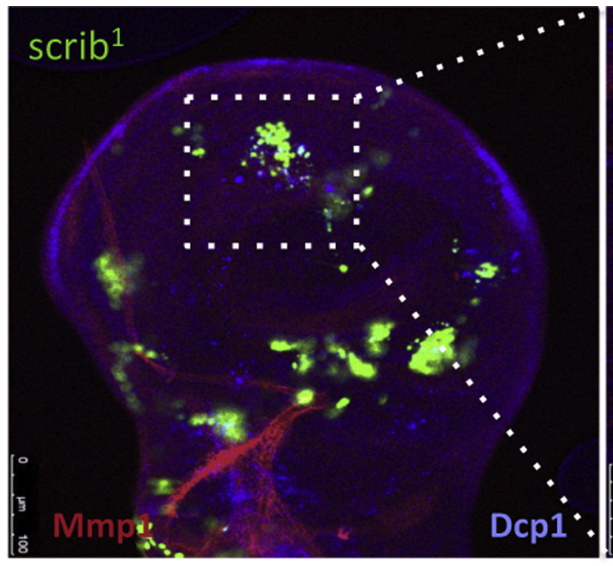

b

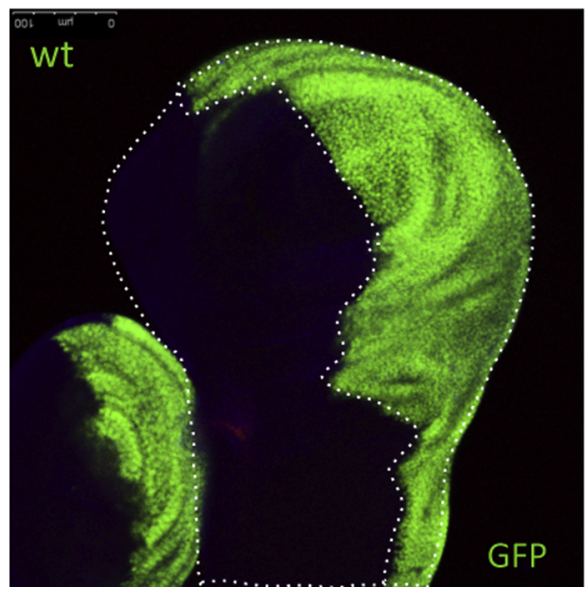

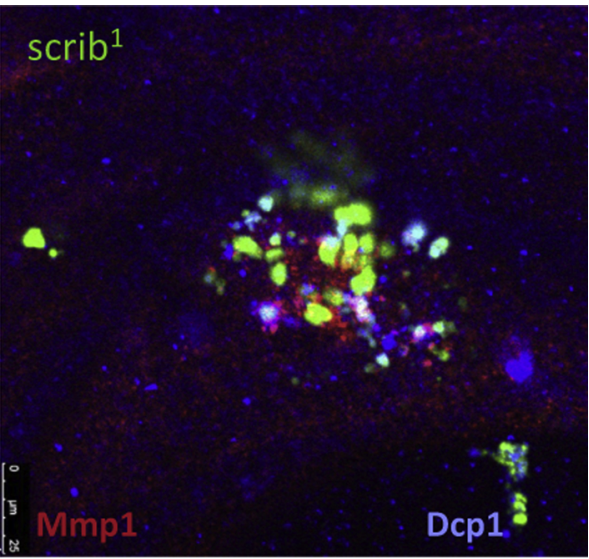

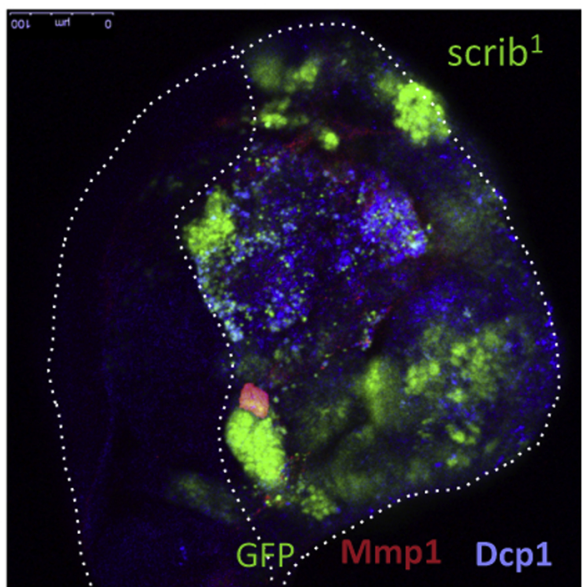

Fig. 2. Distinct behaviour of scrib ${ }^{-}$cells when surrounded by no-tumour cells (a) or when an entire compartment is $s \mathrm{rib}^{-}$(b). The scrib $^{-}$ clone (GFP) in a (magnified in the panel on the right) is being eliminated by cell competition; many cells express the Matrix metalloprotease 1, Mmp1 (red), a JNK target and express the effector caspase Dcp1 (blue). In $\mathbf{b}$ there is the comparison of a wildtype posterior compartment (hh > GFP) with a posterior compartment mutant for scrib. Although the scrib compartment contains high levels of Mmp1 and Dcp1 it still overgrowths reaching a larger size that a normal posterior compartment. 

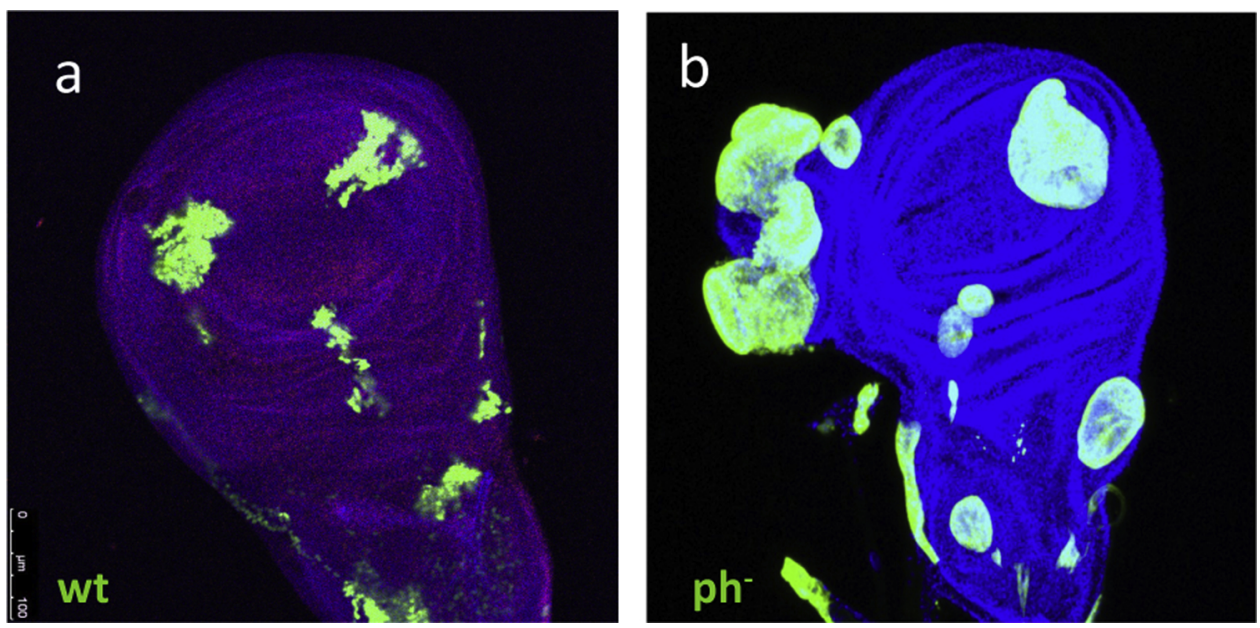

Fig. 3. Comparison of the size and shape of wildtype (a) and polyhomeotic (b) clones in the wing disc. Both sets of clones were induced $72 \mathrm{~h}$ before fixation. Note that some of the polyhomeotic clones are noticeably larger than the wildtype clones and also exhibit very different shape; they tend to be round, showing little indication of interaction cells and integration with the surrounding tissue. In cases they appear to sort out from the disc, like the big clone at the left hand side.

This issue is being intensively investigated in recent years. Some genes of interest have been found by the Igaki group after a screen designed to isolate candidate genes required for the elimination of mutant cells for the oncogenic mutations scribble (scrib) or disc large (dlg). They found that the loss of function of the gene stranded at second (sas), which encodes a ligand protein necessary for axon guidance [52], in winner cells prevents the death of $s c r i b^{-}$loser cells. Moreover, they also identified a specific receptor molecule, PTP10D, which is required in the losers [50]. As a result of the winner/loser interaction, winner cells relocalise the Sas ligand from the apical to the lateral surface of the cells, and the losers relocalise the PTP10D receptor to the same position, thus triggering activation of PTP10D signalling. This causes down regulation of EGFR activity and in turn up regulation of JNK and subsequent apoptosis of loser cells [50]. A similar process occurs during the elimination of mutant cells for vps25, ept or rab5. However, as the authors point out, this mechanism does not appear to function in other cases of cell competition triggered by alteration of Minute or of $d M y c$ levels, raising the possibility that it is specific for elimination of oncogenic cells.

Another factor involved is the secreted protein Serpin5 (Spn5), also necessary to remove $s c r i b{ }^{-}$or $\mathrm{dlg}^{-}$oncogenic cells [53]. Spn5 is secreted by normal epithelial cells and blocks Toll signalling in the oncogenic cells (Ahmad et al., 2019 PNAS), what eventually results in their elimination by the autocrine function of JNK. The functional connection between Sas/PTP10D and Spn5/Toll has not been established.

An interesting observation has been reported by Kucinski [54], pointing out that Minute and mahjong cell competition is associated with oxidative stress in the loser cells, as indicated by overexpression of Nrf2, a major transcription factor involved in the response to oxidative stress. Moreover, we have recently reported a related observation [51]: $s c r i b^{-}$loser clones express high levels of Reactive Oxygen Species (ROS) during the whole time that clones remain alive, about $72 \mathrm{~h}$, also suggesting that oxidative stress is involved in losers elimination.

Since high ROS levels are known to activate JNK [55-59] these findings point out to another possible mechanism of JNK activation based on the idea that the presence of $\mathrm{scrib}^{-}$(or Minute or mahjong) cells may be interpreted as a local stress by the surrounding issue. JNK activation by ROS may become permanent in the cells, for JNK is known to induce the function of the DUAL maturation factor moladietz (mol) that in turn stimulates ROS production, generating a feedback loop [60,61]; see Fig. 1) that maintains JNK activity until the demise of the loser cells. The fact that oxidative stress in loser cells has been shown in at least two different CC scenarios (Minute and mahjong/scrib) suggests that it may be a general feature in the elimination of loser cells.

At this moment there is not an unified model of JNK activation in loser cells that would incorporate the different factors involved, e.g.
Flower, azot, Spz/Toll, Sas/PTP10, Serpin5, ROS. There is also the possibility that different modes of CC may induce JNK by distinct mechanisms.

\section{Incomplete cell competition promotes tumorigenesis}

Even though cell competition exerts a robust anti-tumour function, there are situations in which tumour cells evade cell competition and proceed to form an invasive tumour. As mentioned above, cells mutant for $l g l$, scrib or rab5 are normally eliminated by CC, even if by overexpression of the Ras pathway they have higher proliferation rate than surrounding non-mutant cells [6].

However, we and others $[6,32,62,63]$ reported that discs containing numerous $\mathrm{lgl}^{-} \mathrm{ras}^{\mathrm{V12}}$ (or $\mathrm{scrib}^{-}$ras $^{\mathrm{V12}}$ or rab5 $\mathrm{ras}^{\mathrm{V12}}$ ) clones form large patches of mutant tissue that colonise the entire discs (Fig. 4). However, a detailed study of this finding [6] showed that only discs containing high density of clones were able to form tumour overgrowths; discs with low density failed to produce them. In the latter case the few clones found were small and with many cells in apoptosis.

Based on these results we argued that the failure of cell competition to prevent tumour overgrowths in discs containing numerous clones was due to the merging of neighbour clones. The merging results in large patches of mutant tissue in which many of the inner cells of the patch are beyond the reach of cell competition, which is a short-range phenomenon [21,22]). This group protection mechanism allows tumour cells inside the group to continue proliferating. A scheme of the model is presented in Fig. 4.

Evidence for this mechanism was provided using cells deficient for rab5 activity: isolated rab5 deficient cells are eliminated, but when a group of about 400-500 cells are simultaneously transformed from normal to oncogenic, they survive and develop a tumour outgrowth [33]. It is worth emphasising that cell competition is not abrogated in these conditions; it is restricted to the border of the patches, where the winner/loser interactions take place, as indicated by localisation of cells in apoptosis (illustrated in Fig. 5, see also Fig. 4). A significant fact is that there is continuous turn over of loser cells at the border, as new rab5 cells replace the dead losers. This causes persistent apoptosis, accompanied by persistent JNK activity. Under these conditions the continuous activity of JNK in dying cells results in continuous secretion of mitogenic signals $[64,65]$, what stimulates the proliferation of the tumour cells.

A significant finding from these experiments is that the development of the rab5 tumour outgrowths depends on JNK: in its absence rab5 deficient cells are not eliminated but remain idle and do no over-proliferate [33]. This is a reversal of the normal anti-tumour role of JNK mentioned above and indicates that when CC is not completely effective, the paracrine effect of JNK will stimulate tumour growth 

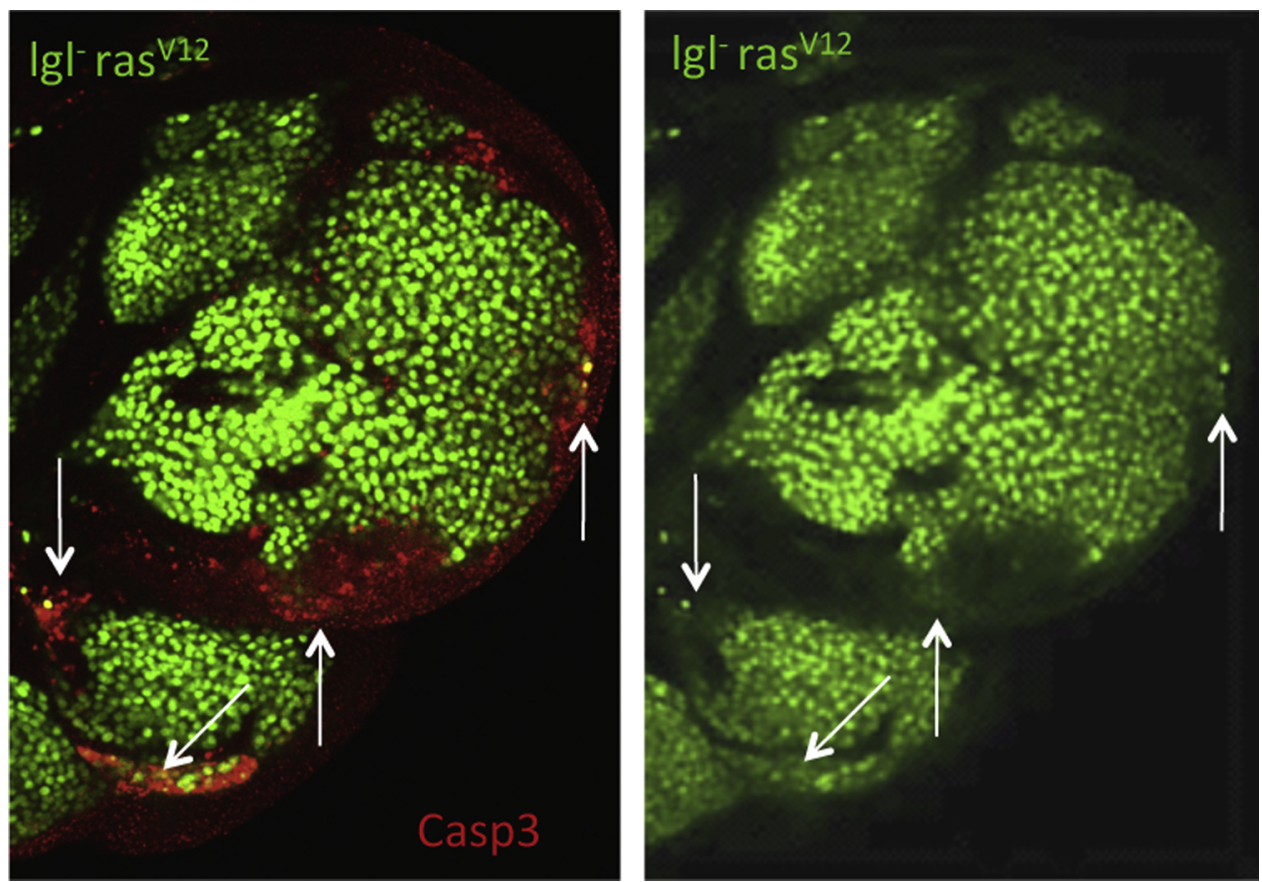

Fig. 4. Wing disc containing large patches of $\mathrm{gl}^{-} \mathrm{ras}^{\mathrm{V12}}$ tissue labelled with GFP that colonise the disc almost entirely. Note the high levels of Caspase activity (red) in the border of the patches and that the dying cells belong to the $\operatorname{lgl}^{-} \mathrm{ras}^{\mathrm{V} 12}$ tissue (arrows).

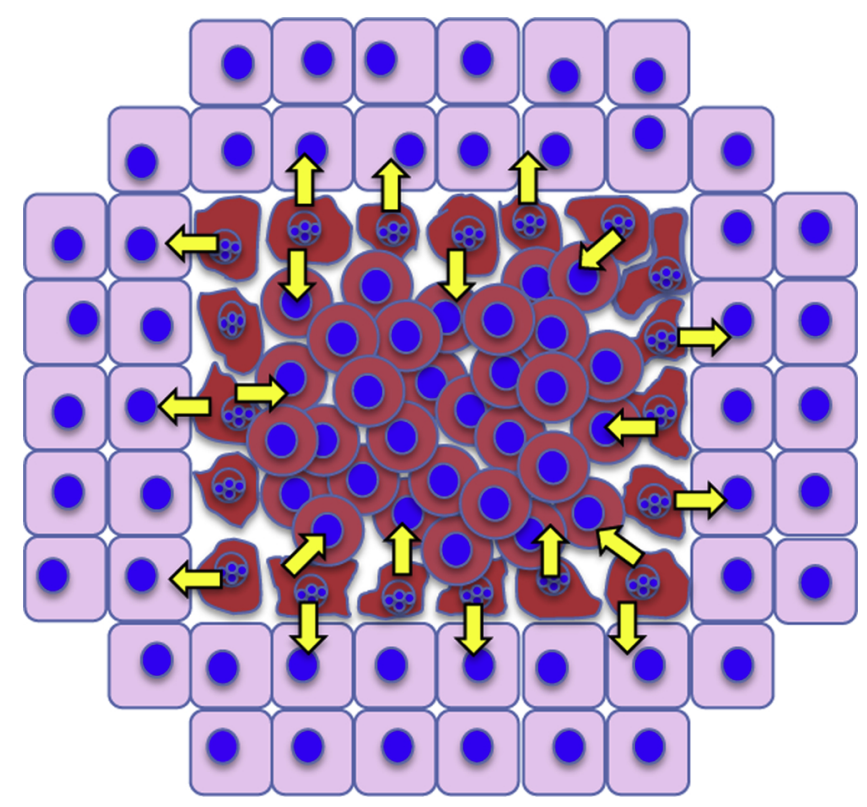

Fig. 5. Scheme of the group protection model of tumorigenesis. Tumour cells are in red, non-tumour ones in pink. Cell nuclei in blue. Because cell competition is a short-range phenomenon tumour cells inside the group are beyond the reach of cell competition; only those at the border interact with non-tumour cells and die by JNK-mediated apoptosis. In the figure they are represented as those with fragmented nuclei. The dying JNK-expressing cells secrete proliferative signals, indicated by the yellow arrows. The signalling emanating from the dying cells can affect cells located either inside or outside the patch. Those inside are tumour cells that are stimulated to divide and contribute to the growth of the tumour. Note that because of the nature of the interaction, once the loser cells at the border are been eliminated, they will be eventually replaced by new ones the resume the interaction.

The fact that rab5 cells can only form a tumour when in a sufficiently large group, may prompt the speculation whether some human cancers may also originate not by individual clones by polyclones in which some cells escape the control mechanisms like CC or others. This kind of mechanism could play a role in the establishment of tumorous growths associated with persistent injuries like for example gastric ulcers. Caused by an infection of Helicobacter pylori, they are associated with inflammation, contain many cells in apoptosis and frequently develop into gastric cancer (reviewed in [66]. It has been shown that mammalian cells in apoptosis secrete proliferative signals [67], and the persistent apoptosis in situations like the gastric ulcer may be a contributing factor in the appearance of the cancer.

\section{Both the tumour-suppressing and the tumour-stimulating functions of cell competition are mediated by JNK activity}

A prime feature of CC is the cell autonomous activation of the JNK pathway in the loser cells that in the majority of the situations leads to their elimination by apoptosis. Thus JNK is the molecular tool used by CC to culling viable but weak, damaged or malignant cells that may appear in animal tissues.

However, JNK is also able to exert a paracrine pro-proliferation function: dying JNK-expressing (loser) cells secrete mitogenic signals that stimulate the proliferation of neighbour cells [64,65], Fig. 1). The paracrine function is inherent to JNK-expressing cells (Fig. 1), but it is normally inconsequential because cells in apoptosis are short-lived. Nevertheless, the removal of oncogenic cells by CC usually takes 2-3 days $[6,33]$, a period during which the cells maintain JNK activity and hence keep secreting mitogenic signals. This result raises the possibility that the paracrine function of JNK may be responsible for the proliferation stimulus of winner cells after the CC interaction $[14,15]$. The finding that tumour overgrowths associated with oncogenic mutations and Ras overexpression [68] requires JNK activity supports this view. Finally, as mentioned above, the pro-proliferative signalling emanating from JNK-expressing cells is also responsible for the tumorous overgrowths found when CC is made ineffective by the group protection mechanism.

The tumour-stimulating activity of the paracrine JNK function becomes evident when the autocrine activity is made ineffective by suppressing the apoptotic machinery [51,61]. In apoptosis-defective cells a transient induction of $\mathrm{JNK}$, by X-radiation or a pulse of P53, is 
translated into persistent JNK activity, which in turn induces the function of the JAK/STAT and Wg and Dpp pathways. The actual mechanism(s) by which these signalling pathways stimulate proliferation is not completely resolved, but the important fact is that JNK has the property of perpetuating its function through a feedback amplification loop that involves ROS and the dual maturation factor moladietz (mol) [61]. This amplification loop was first reported by Khan et al., 2107 when describing the persistence of JNK activity during regeneration of the wing disc.

The two apparently contradictory functions of JNK, pro-apoptotic (anti-tumour) and pro-cell proliferation (tumorigenic), have also been observed in mammalian tissues (reviewed in [69]). Moreover, sustained and elevated levels of JNK activity have been observed in hepatocellular carcinomas both in mice and in human patients [70,71]. It is possible that the some of the mechanisms responsible for tumour overgrowths in Drosophila may also apply to human patients.

\section{Declaration of Competing Interest}

The authors declare no conflict of interest.

\section{Acknowledgements}

We thank the comments, suggestions and support by the members of the laboratory. The experimental work from our group cited in the article has been supported by the Spanish Ministerio de Ciencia e Innovación and by the Fundación Ramón Areces

\section{References}

[1] A.M. Brumby, H.E. Richardson, Using Drosophila melanogaster to map human cancer pathways, Nat. Rev. Cancer 5 (2005) 626-639.

[2] I.K. Hariharan, D. Bilder, Regulation of imaginal disc growth by tumor-suppressor genes in Drosophila, Annu. Rev. Genet. 40 (2006) 335-361.

[3] G. Morata, L. Ballesteros-Arias, Cell competition, apoptosis and tumour development, Int. J. Dev. Biol. 59 (2015) 79-86.

[4] R.N. Salomon, F.R. Jackson, Tumors of testis and midgut in aging flies, Fly (Austin) 2 (2008) 265-268.

[5] L. Menut, T. Vaccari, H. Dionne, J. Hill, G. Wu, et al., A mosaic genetic screen for Drosophila neoplastic tumor suppressor genes based on defective pupation, Genetics 177 (2007) 1667-1677.

[6] J. Menendez, A. Perez-Garijo, M. Calleja, G. Morata, A tumor-suppressing mechanism in Drosophila involving cell competition and the Hippo pathway, Proc. Natl. Acad. Sci. U. S. A. 107 (2010) 14651-14656.

[7] M. Calleja, G. Morata, J. Casanova, Tumorigenic properties of Drosophila Epithelial cells mutant for lethal giant larvae, Dev. Dyn. 245 (2016) 834-843.

[8] I. Martincorena, A. Roshan, M. Gerstung, P. Ellis, P. Van Loo, et al., Tumor evolution. High burden and pervasive positive selection of somatic mutations in normal human skin, Science 348 (2015) 880-886.

[9] G. Morata, P. Ripoll, Minutes: mutants of drosophila autonomously affecting cell division rate, Dev. Biol. 42 (1975) 211-221.

[10] S.J. Marygold, J. Roote, G. Reuter, A. Lambertsson, M. Ashburner, et al., The ribosomal protein genes and Minute loci of Drosophila melanogaster, Genome Biol. 8 (2007) R216.

[11] J.Y. Lee, M.S. Kim, J.E. Ju, M.S. Lee, N. Chung, et al., Simvastatin enhances the radiosensitivity of p53deficient cells via inhibition of mouse double minute 2 homolog, Int. J. Oncol. 52 (2018) 211-218.

[12] R. Bohni, J. Riesgo-Escovar, S. Oldham, W. Brogiolo, H. Stocker, et al., Autonomous control of cell and organ size by CHICO, a Drosophila homolog of vertebrate IRS1-4, Cell 97 (1999) 865-875.

[13] L.A. Johnston, D.A. Prober, B.A. Edgar, R.N. Eisenman, P. Gallant, Drosophila myc regulates cellular growth during development, Cell 98 (1999) 779-790.

[14] C. de la Cova, M. Abril, P. Bellosta, P. Gallant, L.A. Johnston, Drosophila myc regulates organ size by inducing cell competition, Cell 117 (2004) 107-116.

[15] E. Moreno, K. Basler, dMyc transforms cells into super-competitors, Cell 117 (2004) $117-129$.

[16] E. Moreno, Is cell competition relevant to cancer? Nat. Rev. Cancer 8 (2008) $141-147$.

[17] G. Kolahgar, S.J. Suijkerbuijk, I. Kucinski, E.Z. Poirier, S. Mansour, et al., Cell competition modifies adult stem cell and tissue population dynamics in a JAKSTAT-Dependent manner, Dev. Cell 34 (2015) 297-309.

[18] P. Simpson, G. Morata, Differential mitotic rates and patterns of growth in compartments in the Drosophila wing, Dev. Biol. 85 (1981) 299-308.

[19] T. Adachi-Yamada, M.B. O'Connor, Morphogenetic apoptosis: a mechanism for correcting discontinuities in morphogen gradients, Dev. Biol. 251 (2002) 74-90.

[20] M. Milan, L. Perez, S.M. Cohen, Short-range cell interactions and cell survival in the
Drosophila wing, Dev. Cell 2 (2002) 797-805.

[21] F.A. Martin, A. Perez-Garijo, G. Morata, Apoptosis in Drosophila: compensatory proliferation and undead cells, Int. J. Dev. Biol. 53 (2009) 1341-1347.

[22] R. Levayer, B. Hauert, E. Moreno, Cell mixing induced by myc is required for competitive tissue invasion and destruction, Nature 524 (2015) 476-480.

[23] E.R. Oliver, T.L. Saunders, S.A. Tarle, T. Glaser, Ribosomal protein L24 defect in belly spot and tail (Bst), a mouse minute, Development 131 (2004) 3907-3920.

[24] M. Oertel, A. Menthena, Y.Q. Chen, D.A. Shafritz, Properties of cryopreserved fetal liver stem/progenitor cells that exhibit long-term repopulation of the normal rat liver, Stem Cells 24 (2006) 2244-2251.

[25] C. Claveria, G. Giovinazzo, R. Sierra, M. Torres, Myc-driven endogenous cell competition in the early mammalian embryo, Nature 500 (2013) 39-44.

[26] M. Sancho, A. Di-Gregorio, N. George, S. Pozzi, J.M. Sanchez, et al., Competitive interactions eliminate unfit embryonic stem cells at the onset of differentiation, Dev. Cell 26 (2013) 19-30.

[27] S.J. Ellis, N.C. Gomez, J. Levorse, A.F. Mertz, Y. Ge, et al., Distinct modes of cell competition shape mammalian tissue morphogenesis, Nature 569 (2019) 497-502.

[28] G. Struhl, K. Basler, Organizing activity of wingless protein in Drosophila, Cell 72 (1993) 527-540.

[29] T. Xu, G.M. Rubin, Analysis of genetic mosaics in developing and adult Drosophila tissues, Development 117 (1993) 1223-1237.

[30] T. Igaki, J.C. Pastor-Pareja, H. Aonuma, M. Miura, T. Xu, Intrinsic tumor suppression and epithelial maintenance by endocytic activation of Eiger/TNF signaling in Drosophila, Dev. Cell 16 (2009) 458-465.

[31] Y. Tamori, C.U. Bialucha, A.G. Tian, M. Kajita, Y.C. Huang, et al., Involvement of Lgl and Mahjong/VprBP in cell competition, PLoS Biol. 8 (2010) e1000422.

[32] C.L. Chen, M.C. Schroeder, M. Kango-Singh, C. Tao, G. Halder, Tumor suppression by cell competition through regulation of the Hippo pathway, Proc. Natl. Acad. Sci. U. S. A. 109 (2012) 484-489.

[33] L. Ballesteros-Arias, V. Saavedra, G. Morata, Cell competition may function either as tumour-suppressing or as tumour-stimulating factor in Drosophila, Oncogene 33 (2014) 4377-4384.

[34] M. Barbacid, Ras genes, Annu. Rev. Biochem. 56 (1987) 779-827.

[35] J.V. Beira, J. Torres, R. Paro, Signalling crosstalk during early tumorigenesis in the absence of Polycomb silencing, PLoS Genet. 14 (2018) e1007187.

[36] Y. Jiang, M. Seimiya, T.B. Schlumpf, R. Paro, An intrinsic tumour eviction mechanism in Drosophila mediated by steroid hormone signalling, Nat. Commun. 9 (2018) 3293.

[37] J. Torres, R. Monti, A.L. Moore, M. Seimiya, Y. Jiang, et al., A switch in transcription and cell fate governs the onset of an epigenetically-deregulated tumor in Drosophila, Elife (2018) 7

[38] M. Norman, K.A. Wisniewska, K. Lawrenson, P. Garcia-Miranda, M. Tada, et al., Loss of Scribble causes cell competition in mammalian cells, J. Cell. Sci. 125 (2012) $59-66$.

[39] M. Kajita, K. Sugimura, A. Ohoka, J. Burden, H. Suganuma, et al., Filamin acts as a key regulator in epithelial defence against transformed cells, Nat. Commun. 5 (2014) 4428

[40] V.C. Martins, K. Busch, D. Juraeva, C. Blum, C. Ludwig, et al., Cell competition is a tumour suppressor mechanism in the thymus, Nature 509 (2014) 465-470.

[41] S. Brown, C.M. Pineda, T. Xin, J. Boucher, K.C. Suozzi, et al., Correction of aberrant growth preserves tissue homeostasis, Nature 548 (2017) 334-337.

[42] C.K. Yao, Y.Q. Lin, C.V. Ly, T. Ohyama, C.M. Haueter, et al., A synaptic vesicleassociated $\mathrm{Ca} 2+$ channel promotes endocytosis and couples exocytosis to endocytosis, Cell 138 (2009) 947-960.

[43] C. Rhiner, J.M. Lopez-Gay, D. Soldini, S. Casas-Tinto, F. A. Martin, et al., Flower forms an extracellular code that reveals the fitness of a cell to its neighbors in Drosophila, Dev. Cell 18 (2010) 985-998.

[44] M.M. Merino, C. Rhiner, J.M. Lopez-Gay, D. Buechel, B. Hauert, et al., Elimination of unfit cells maintains tissue health and prolongs lifespan, Cell 160 (2015) $461-476$

[45] S.N. Meyer, M. Amoyel, C. Bergantinos, C. de la Cova, C. Schertel, et al., An ancient defense system eliminates unfit cells from developing tissues during cell competition, Science 346 (2014) 1258236.

[46] L. Alpar, C. Bergantinos, L.A. Johnston, Spatially restricted regulation of Spatzle/ Toll signaling during cell competition, Dev. Cell 46 (2018) 706-719 e705.

[47] F. Germani, D. Hain, D. Sternlicht, E. Moreno, K. Basler, The Toll pathway inhibits tissue growth and regulates cell fitness in an infection-dependent manner, Elife (2018) 7.

[48] E. Moreno, K. Basler, G. Morata, Cells compete for decapentaplegic survival factor to prevent apoptosis in Drosophila wing development, Nature 416 (2002) 755-759.

[49] S. Ohsawa, K. Sugimura, K. Takino, T. Xu, A. Miyawaki, et al., Elimination of oncogenic neighbors by JNK-mediated engulfment in Drosophila, Dev. Cell 20 (2011) 315-328.

[50] M. Yamamoto, S. Ohsawa, K. Kunimasa, T. Igaki, The ligand Sas and its receptor PTP10D drive tumour-suppressive cell competition, Nature 542 (2017) 246-250.

[51] N. Pinal, M. Calleja, G. Morata, Pro-apoptotic and pro-proliferation functions of the JNK pathway of Drosophila: roles in cell competition, tumorigenesis and regeneration, Open Biol. 9 (2019) 180256.

[52] H.K. Lee, A. Cording, J. Vielmetter, K. Zinn, Interactions between a receptor tyr osine phosphatase and a cell surface ligand regulate axon guidance and glial-neuronal communication, Neuron 78 (2013) 813-826.

[53] M. Katsukawa, S. Ohsawa, L. Zhang, Y. Yan, T. Igaki, Serpin facilitates tumorsuppressive cell competition by blocking toll-mediated yki activation in Drosophila, Curr. Biol. 28 (2018) 1756-1767 e1756.

[54] I. Kucinski, M. Dinan, G. Kolahgar, E. Piddini, Chronic activation of JNK JAK/STAT and oxidative stress signalling causes the loser cell status, Nat. Commun. 8 (2017) 
136.

[55] M. Morey, M. Corominas, F. Serras, DIAP1 suppresses ROS-induced apoptosis caused by impairment of the selD/sps1 homolog in Drosophila, J. Cell. Sci. 116 (2003) 4597-4604.

[56] P. Santabarbara-Ruiz, M. Lopez-Santillan, I. Martinez-Rodriguez, A. Binagui-Casas, L. Perez, et al., ROS-induced JNK and p38 signaling is required for unpaired cytokine activation during Drosophila regeneration, PLoS Genet. 11 (2015) e1005595.

[57] C.W. Wang, A. Purkayastha, K.T. Jones, S.K. Thaker, U. Banerjee, In vivo genetic dissection of tumor growth and the Warburg effect, Elife (2016) 5.

[58] E. Perez, J.L. Lindblad, A. Bergmann, Tumor-promoting function of apoptotic caspases by an amplification loop involving ROS, macrophages and JNK in Drosophila, Elife (2017) 6.

[59] N. Diwanji, A. Bergmann, An unexpected friend - ROS in apoptosis-induced compensatory proliferation: implications for regeneration and cancer, Semin. Cell Dev. Biol. 80 (2018) 74-82.

[60] A.R. Brock, M. Seto, R.K. Smith-Bolton, Cap-n-Collar promotes tissue regeneration by regulating ROS and JNK signaling in the Drosophila melanogaster wing imaginal disc, Genetics 206 (2017) 1505-1520.

[61] N. Pinal, M. Martin, I. Medina, G. Morata, Short-term activation of the Jun Nterminal kinase pathway in apoptosis-deficient cells of Drosophila induces tumorigenesis, Nat. Commun. 9 (2018) 1541.

[62] A.M. Brumby, H.E. Richardson, Scribble mutants cooperate with oncogenic Ras or Notch to cause neoplastic overgrowth in Drosophila, EMBO J. 22 (2003)
5769-5779.

[63] R.A. Pagliarini, T. Xu, A genetic screen in Drosophila for metastatic behavior, Science 302 (2003) 1227-1231.

[64] A. Perez-Garijo, F.A. Martin, G. Morata, Caspase inhibition during apoptosis causes abnormal signalling and developmental aberrations in Drosophila, Development 131 (2004) 5591-5598.

[65] H.D. Ryoo, T. Gorenc, H. Steller, Apoptotic cells can induce compensatory cell proliferation through the JNK and the Wingless signaling pathways, Dev. Cell 7 (2004) 491-501.

[66] L.E. Wroblewski, R.M. Peek Jr., K.T. Wilson, Helicobacter pylori and gastric cancer: factors that modulate disease risk, Clin. Microbiol. Rev. 23 (2010) 713-739.

[67] J.X. Wang, J.Q. Jiao, Q. Li, B. Long, K. Wang, et al., miR-499 regulates mitochon drial dynamics by targeting calcineurin and dynamin-related protein-1, Nat. Med. 17 (2011) 71-78.

[68] M. Wu, J.C. Pastor-Pareja, T. Xu, Interaction between Ras(V12) and scribbled clones induces tumour growth and invasion, Nature 463 (2010) 545-548.

[69] E.F. Wagner, A.R. Nebreda, Signal integration by JNK and p38 MAPK pathways in cancer development, Nat. Rev. Cancer 9 (2009) 537-549.

[70] T. Sakurai, S. Maeda, L. Chang, M. Karin, Loss of hepatic NF-kappa B activity enhances chemical hepatocarcinogenesis through sustained c-Jun N-terminal kinase 1 activation, Proc. Natl. Acad. Sci. U. S. A. 103 (2006) 10544-10551.

[71] Q. Chang, Y. Zhang, K.J. Beezhold, D. Bhatia, H. Zhao, et al., Sustained JNK1 activation is associated with altered histone $\mathrm{H} 3$ methylations in human liver cancer, $\mathrm{J}$ Hepatol. 50 (2009) 323-333. 